ARTÍCULO ORIGINAL

\title{
Monepantel y control de Haemonchus contortus en ovinos. Evaluación de la eficacia luego de cuatro años de uso en condiciones de refugio reducido.
}

\author{
Torrents $\mathrm{J}^{1}$, Suarez Archilla $\mathrm{G}^{2}$, Anziani $\mathrm{OS}^{2,3^{*}}$ \\ Facultad de Ciencias Veterinarias, Universidad Nacional del Litoral, Santa Fe, Argentina. \\ EEA INTA Rafaela, Santa Fe, Argentina \\ Facultad Ciencias Agropecuarias, Universidad Católica de Córdoba, Córdoba, Argentina \\ * Correspondencia: OS Anziani. EEA INTA Rafaela, Ruta 34 Km 2272300 Rafaela Santa Fe, Santa Fe, Argentina. E-mail: \\ anziani.oscar@inta.gob.ar
}

Recibido: 1 Abril 2016. Aceptado: 14 Abril 2016. Disponible en línea: 15 Abril 2016.

Editor: S. Nava

RESUMEN. La resistencia de los nematodes ovinos a los antihelmínticos está generalizada en el centro-norte de la Argentina y la mayor preocupación actual es el aumento y dispersión de Haemonchus contortus portando alelos con resistencia múltiple. Actualmente los únicos antihelmínticos de amplio espectro sin documentación de resistencia en nuestro país son el monepantel y derquantel. Con la primera de estas drogas ya se han registrado casos de resistencia en Uruguay y Brasil por lo que es crítica la información actualizada sobre la actividad en el campo de estos antihelmínticos. Este estudio informa sobre la eficacia del monepantel luego de cuatro años de uso de esta droga sobre un aislamiento de $H$. contortus con resistencia múltiple (lactonas macrociclicas, closantel, levamisol y benzimidazoles) y bajo condiciones de refugio reducido. Se realizaron dos experiencias A y B (años 2014 y 2016 respectivamente) con corderos naturalmente parasitados y seleccionados en función del número de huevos por gramo de heces ( $\geq$ 250) los que fueron tratados con monepantel $(2,5 \mathrm{mg} / \mathrm{Kg}$ oral). El test de reducción en el conteo de huevos post tratamiento comparando diversas fórmulas indicó que en ambas experiencias, la droga mantuvo una eficacia mayor al $97 \%$ y que permanece activa para controlar este aislamiento de $\mathrm{H}$. contortus luego de cuatro años de uso y bajo las particulares condiciones en las que se realizaron estos estudios.

SUMMARY. Monepatel and the control of Haemonchus contortus in sheep. Efficacy assessment after four years of use in reduced refugia. Resistance to anthelmintics in sheep nematode is widespread in central-northern Argentina and current major concern is the rise and spread of Haemonchus contortus carrying multiple resistance alleles. Currently, monepantel and derquantel are the only broad-spectrum anthelmintics without documentation of resistance in our country. With the first of these drugs, have already been registered cases of resistance in Uruguay and Brazil so it is critical update on the activity in the field of these anthelmintics. This study aims to test the effectiveness of monepantel after four years of use of this drug on an isolation of $\mathrm{H}$. contortus with multiple resistance (macrocyclic lactones, closantel, levamisole and benzimidazole) and under conditions of reduced refugia. Two experiences, A and B (2014 and 2016 respectively), were carried out with lambs naturally parasitized and selected by the number of eggs per gram of feces $(\geq 250)$. In both experiences, the test of reduction in egg counts after treatment with monepantel $(2.5 \mathrm{mgr} / \mathrm{Kg}$ orally) comparing various formulas indicated that the drug remained with efficacy greater than $97 \%$ after four years of use to control this isolation of $H$. contortus under the field conditions of these studies.

Palabras clave: Haemonchus, ovinos, monepatel, Argentina.

Key words: Haemonchus, sheep, monepatel, Argentina.

\section{Introducción}

Los nematodes gastrointestinales constituyen actualmente y desde una perspectiva global, la causa más importante de pérdidas productivas en los ovinos y en este contexto el género Haemonchus es considerado el de mayor patogenicidad e impacto económico en todo el mundo (Waller, 2003; Bessier, 2007). La administración de antihelmínticos constituye la principal herramienta para el control aunque el desarrollo creciente de la resistencia a estas drogas está comprometiendo seriamente la sustentabilidad de esta alternativa (Kaplan, 2004).

La presión de selección que ejerce una droga antihelmíntica depende del modo que la misma es utilizada en el campo. Además del uso masivo y frecuente, otra forma de presión de selección es la aplicación de antihelmínticos cuando las posibilidades de re-infección son bajas (refugio mínimo). Se denomina refugio a las poblaciones de nematodes que no son alcanzadas 
por la administración de antihelmínticos como por ejemplo los huevos y estados larvales en pasturas y los adultos en animales que no son tratados (VanWyk, 2001). Esta sub-población en refugio es crítica para acelerar o disminuir la aparición de resistencia (a mayor refugio menor desarrollo de la resistencia) ya que representa un reservorio de parásitos susceptibles que pueden reproducirse con los que sobreviven al trata-miento "diluyendo" así los alelos resistentes (Van Wyk, 2001; Dobson et al, 2011; Leathwick, 2012).

En nuestro país, las fallas de eficacia en el control de los nematodes ovinos es un fenómeno generalizado en todo el centro y norte (Romero et al, 2013; Anziani \& Fiel, 2015) y la mayor preocupación actual es el aumen-to y dispersión de aislamientos con resistencia a varios antihelmínticos. Así por ejemplo aislamientos de Hae-monchus contortus con resistencia múltiple a dos, tres y cuatro drogas con diferentes modos de acción han sido informados mayormente en el litoral (Romero et al, 2007) pero también en provincias del área central de la Argentina como Buenos Aires (Entrocasso et al, 2008; Steffan et al, 2011) y Santa Fe (Anziani \& Muchiut 2014). En el presente las únicas drogas antihelmínticas de amplio espectro con los cuales no han sido documen-tados fenómenos de resistencia son el monepantel y el derquantel, dos grupos químicos introducidas en el mercado veterinario argentino para uso exclusivo en ovinos en el 2011 y el 2015 respectivamente (Steffan et al, 2011; Fiel C., comunicación personal). Sin embargo, existe también preocupación sobre el futuro de estos nuevos antihelmínticos ya que, en el primero de los casos y luego de solo dos a tres años de uso se han sido informado casos de resistencia sobre varios géneros de parásitos gastrointestinales en Nueva Zelandia, Austra-lia, Holanda, Uruguay y Brasil (Scott et al, 2013; Sales \& Love, 2014; Van den Brom et al, 2015; Mederos et al, 2014; Cintra et al, 2016).

La viabilidad de la producción ovina del centro y norte de la Argentina depende fuertemente de los antihelmínticos y por lo tanto es indispensable contar con información actualizada sobre la eficacia de las drogas que aún mantienen actividad contra los nematodes gastrointestinales para identificar en forma temprana la aparición de resistencia y evitar las pérdidas productivas resultantes. En este contexto, el objetivo del presente trabajo es comunicar la eficacia del monepantel luego de cuatro años de uso de esta droga sobre un aislamiento de $\mathrm{H}$. contortus con resistencia múltiple (lactonas macrociclicas, closantel, levamisol y benzi-midazoles) y bajo condiciones consideradas como de refugio reducido o mínimo.

\section{Materiales y métodos}

a) Localización de las experiencias, antecedentes y animales utilizados: el estudio se desarrolló en la EEA INTA Rafaela durante los meses de Octubre-Noviem- bre y Enero-Febrero de los años 2014 y 2016 experiencias A y B (respectivamente). Como animales experimentales se utilizaron 29 corderos de 3 a 6 meses de edad de raza Pampinta pertenecientes a una majada de la EEA INTA Rafaela y mantenida en esta institución con fines experimentales. Todos los animales de la majada permanecieron (durante al menos los últimos seis años) en condiciones de pastoreo continuo sobre una misma pastura natural (generando condiciones de alta contaminación de la misma y reinfección permanente) y suplementados con heno de alfalfa. En la majada existen desde el año 2012 antecedentes de nematodes gastrointestinales $(H$. contortus) con resistencia múltiple o simultánea a lactonas macrocíclicas, levamisol, benzimidazoles y closantel (Anziani y Muchiut, 2013). Esta situación determinó que hacia mediados del 2012 y hasta el 2015 inclusive, el control de los nematodes gastrointestinales se basara en la aplicación exclusiva de monepantel ("Zolvix"® ${ }^{\oplus}$, Zoetis). El número de tratamientos anuales en la categoría de corderos fue de tres en el año 2012 a cuatro en el período 2013-2015 y desde aproximadamente los dos meses hasta el año de edad (frecuencia entre 52 a 85 días) mientras que el resto de los integrantes de la majada fueron tratados con dos tratamientos anuales durante este período.

b) Diseño de la experiencia, tratamientos y determinaciones parasitológicas: La eficacia de la droga se determinó a través del test de reducción en el conteo de huevos (TRCH). Entre los días -1 y -2 de cada una de las experiencias, los corderos fueron pesados, identificados con caravanas y se tomaron muestras de materia fecal para determinar el número de huevos de nematodes por gramo de heces $(\mathrm{hpg})$ mediante la técnica de Mc Master modificada (Roberts y O' Sullivan, 1949). Se seleccionaron para ambas experiencias animales con valores del hpg $\geq 250$. Alícuotas de materia fecal fueron obtenidas para la realización de coprocultivos y recuperación de larvas de tercer estadío a través de un aparato de Baermann, para identificación de los géneros de nematodes intervinientes. En el día 0 se realizaron los tratamientos con monepantel por vía oral a la dosis de $2,5 \mathrm{mg} / \mathrm{Kg}$ de peso vivo y en los días 9 y 14 post tratamiento (experiencias A y B respectivamente) se tomaron nuevas muestras de materia fecal para establecer el TRCH y determinar la eficacia para esta droga. Luego de los tratamientos, los corderos permanecieron sobre la misma pastura (descripta en el punto a) y las determinaciones del hpg continuaron hasta que los mismos retornaran a los valores pre-tratamiento.

c) Análisis de datos y criterios para la evaluación de susceptibilidad y/o resistencia: Para el TRCH se siguieron las recomendaciones generales de la World Association of Veterinary Parasitology (Coles et al 1992), considerando el porcentaje de reducción del hpg y los límites de los intervalos de confianza al $95 \%$. Los tratamientos fueron considerados efectivos si el TRCH 
y el límite superior del intervalo de confianza fueron iguales o superiores al $95 \%$ y el límite menor de este último igual o superior al $90 \%$. En la experiencia " $\mathrm{A}$ " además del grupo tratado se utilizó un grupo control sin tratamiento mientras que en la experiencia $B$ se prescindió del mismo y se compararon exclusivamente los valores del hpg pre- y pos-tratamiento de los mismos animales. En general, actualmente se considera que estas últimas formas de evaluar la actividad de las drogas sobre la oviposición (hpg) de las hembras de nematodes, puede producir resultados tan consistentes como cuando se utilizan animales controles (Dobson et al, 2012) disminuyendo costos y tiempo así como potenciales objeciones (bienestar animal) asociadas a la ausencia de tratamientos en animales experimentales parasitados. En términos de especificidad en ambos métodos es del $100 \%$ mientras que en la sensibilidad del TRCH no existen diferencias significativas $(0,92$ vs 0,96$)$ para presencia o ausencia de controles sin trata-miento (McKenna, 2006).

Las fórmulas empleadas en los TRCH del presente traba-jo fueron las siguientes:

1) $\mathrm{TRCH}=100 \times(1-[\mathrm{T} 2 / \mathrm{C} 2])$. Coles et.al 1992.

2) $\mathrm{RCH}=100 \times(1-[\mathrm{T} 2 / \mathrm{T} 1] \times[\mathrm{C} 1 / \mathrm{C} 2])$ Dash et.al, 1988.

3) $\mathrm{RCH}=100 \times(1-[\mathrm{T} 2 / \mathrm{T} 1])$ McKenna 2006.

4) $\mathrm{RCH}=100 \times(1-p)$. donde $p=x / n$. Donde " $x$ " es la sumatoria de los huevos postratamiento $y$ " $n$ " es la sumatoria de los huevos pretratamiento. Dobson 2012.

5) $\mathrm{RCH}(1 / \mathrm{n}) \times \Sigma(100 \times(1-\mathrm{Ti} 2 / \mathrm{Ti} 1))$. Los valores de $\mathrm{Ti}$ son los valores de hpg individuales, realizando una reducción individual. Esta fórmula está basada en el promedio de la sumatoria de las reducciones individuales. Cabaret 2004.

Los valores de T y C indican la media aritmética del hpg en el grupo tratado o control mientras que 1 y 2 indican si estos valores son pre o post tratamiento respectivamente.

\section{Resultados y discusión}

Las larvas obtenidas en los coprocultivos pre-tratamiento de ambas experiencias mostró la presencia casi exclusiva de Haemonchus contortus (> 98\%) y el resto perteneciente al género Trichostrongylus spp. Los tratamientos resultaron en reducciones del hpg del $100 \%$ para las experiencias A y B, indicando que este aislamiento de Haemonchus contortus con resistencia a múltiples antihelmínticos continúa siendo altamente susceptible al monepantel luego de cuatro años de uso continuo en esta población parasitaria. Una síntesis de los resultados sobre la eficacia de esta droga es presentada en la Tabla 1. Estos valores de eficacia no difieren de lo observado en el año 2012 cuando el primer tratamiento con la misma dosis de monepantel y en esta misma majada resultó en un TRCH $>99,8 \%$ (EEA INTA Rafaela, datos no publicados). Como se indicara anteriormente los animales permanecen desde hace años siempre sobre la misma superficie de pastoreo sin ingreso de otros ovinos (a excepción de dos reproductores machos ingresados en 2013) y los tratamientos habían comprendido en los cuatro años previos a la totalidad de los corderos de cada cohorte y a sus madres. En este contexto las condiciones de refugio desde la instauración del primer tratamiento hasta el presente pueden ser consideradas como reducidas y potencialmente haber permitido ventajas competitivas para el establecimiento de Haemonchus sp. resistentes. No obstante, nuestras observaciones demuestran que la eficacia de la droga (aunque medida con un test de baja sensibilidad como el TRCH) permanece como muy alta. Lamentablemente no se encuentran disponibles otros métodos de mayor sensibilidad y el TRCH continúa siendo considerado como el gold standard para detectar susceptibilidad/resistencia bajo condiciones de campo (Anziani \& Fiel, 2015).

Hasta el presente, la alta eficacia del monepantel luego de casi cuatro años consecutivos de uso (18 tratamientos) se encuentran en línea con los resultados informados originalmente con el ingreso de la droga al mercado veterinario argentino (Stefan et al, 2011). Al contrario de lo observado en el presente trabajo y utilizando el mismo test in vivo, en otros países como Holanda por ejemplo, se han desarrollado aislamientos de Haemonchus sp. resistentes al monepantel luego de solo tres años de uso y con una frecuencia similar o inferior de tratamientos (Van den Brom, 2015). Así mismo en Uruguay (Mederos et al, 2014) se informó sobre el desarrollo de Haemonchus sp. resistentes al monepantel luego de tres años y aún con antecedentes del uso selectivo de la droga (método Famacha) lo cual resulta en mayor refugio que los tratamientos masivos como los que se habían empleado en la majada de nuestra experiencia.

Con respecto al período de reaparición de huevos, en ambas experiencias quedo confirmada la reducida persistencia de la droga (Stein et al, 2011). Bajo las condiciones de re-infección continua de nuestra experiencia, aproximadamente a los 30 días post tratamiento se observaron valores promedios del hpg iguales o mayores a los observados al momento del mismo. Una síntesis de estas observaciones es presentada en la Figura 1.

En conclusión, en esta majada parasitada por Haemonchus contortus con resistencia a múltiples antihelmínticos (lactonas macrocíclicas, benzimidazoles, levamisol y closantel) la eficacia del monepantel evaluada a través del TRCH se mantiene 
luego de casi cuatro años de uso. En este contexto la droga permanece con un interesante potencial para el control de nematodes gastrointestinales de los ovinos en el área central de la Argentina, especialmente en establecimientos con antecedentes de resistencia antihelmíntica. Sin embargo y donde las condiciones de susceptibilidad parasitaria lo permitan, la droga de- bería siempre ser utilizada en rotación con otros antihelmínticos tal como ha sido recomendado enfáticamente (Kaminsky et al, 2013) a los fines de evitar el rápido desarrollo de resistencia.

Tabla 1. Eficacia de monepantel $(2,5 \mathrm{mg} / \mathrm{kg}$ oral) sobre un asilamiento de Haemonchus contortus con resistencia múltiple en corderos naturalmente parasitados. Promedios de los hpg (rango) y porcentaje en el TRCH comparando diferentes fórmulas que utilizan o prescinden de animales controles.

\begin{tabular}{|c|c|c|c|c|c|c|c|c|c|}
\hline \multirow{4}{*}{$\begin{array}{c}\text { Experiencia } \\
\text { A }\end{array}$} & \multirow[t]{2}{*}{ GRUPO } & \multirow[t]{2}{*}{ N } & \multirow{2}{*}{$\begin{array}{c}\text { HPG } \\
\text { Pre-tratam. }\end{array}$} & \multirow{2}{*}{$\begin{array}{c}\text { HPG } \\
\text { Pos-tratam. }\end{array}$} & \multicolumn{5}{|c|}{ TRCH (\%) } \\
\hline & & & & & $\begin{array}{c}\text { formula } \\
1\end{array}$ & $\begin{array}{c}\text { formula } \\
2\end{array}$ & $\begin{array}{c}\text { formula } \\
3\end{array}$ & formula 4 & formula 5 \\
\hline & tratamiento & 10 & $\begin{array}{c}1263 \\
(240-3390)\end{array}$ & $\begin{array}{c}16 \\
(0-80)\end{array}$ & 98,8 & 98,8 & 98,7 & 99,5 & 99,5 \\
\hline & control & 8 & $\begin{array}{c}1181 \\
(210-3600)\end{array}$ & $\begin{array}{c}1381 \\
(510-3820)\end{array}$ & & & & & \\
\hline $\begin{array}{c}\text { Experiencia } \\
\text { B }\end{array}$ & & 11 & $\begin{array}{c}1841 \\
(360-4470)\end{array}$ & $\begin{array}{c}3,7 \\
(0-40)\end{array}$ & $\begin{array}{l}{ }^{*} \text { no } \\
\text { corresp. }\end{array}$ & $\begin{array}{l}{ }^{*} \text { no } \\
\text { corresp. }\end{array}$ & 99,8 & 99,8 & 99,8 \\
\hline
\end{tabular}

${ }^{*}$ no corresponde ya que fórmulas 1 y 2 utilizan grupos controles.

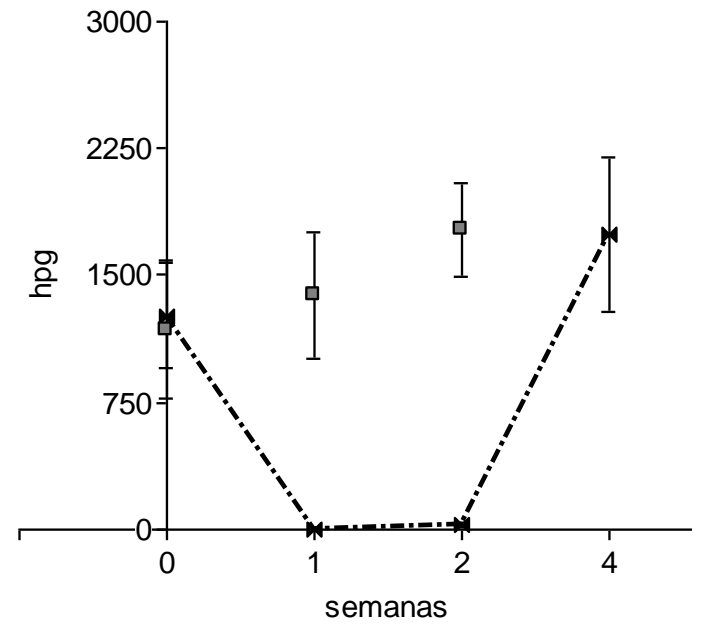

--A- Tratados $\square$ Controles

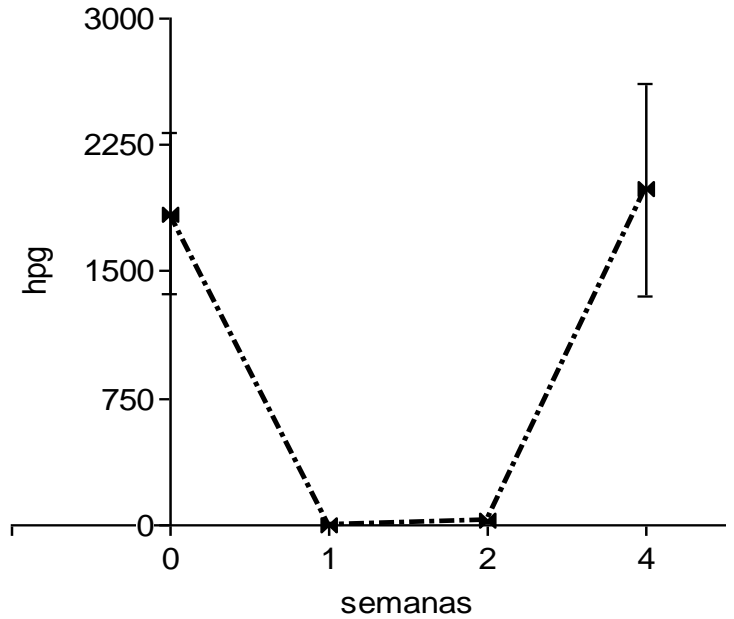

$\boldsymbol{- \cdots - 1}$ Tratados

Figura 1. Evolución del promedio del hpg y error standard en corderos naturalmente parasitados por Haemonchus contortus con resistencia múltiple y tratados con monepantel $(2,5 \mathrm{mg} / \mathrm{Kg}$ oral) en las experiencias $A$ (izquierda) y $B$ respectivamente (derecha).

\section{Agradecimientos}

Al convenio INTA-AUDEAS-CONADEV (CIAC 940143), al PE INTA (1115055) y a la secretaría investigación de la Universidad Católica de Córdoba por la financiación del presente trabajo.

\section{References}

Anziani OS, Fiel CA. 2015. Resistencia a los antihelmínticos en nematodos que parasitan a los rumiantes en la Argentina. RIA. 41: 34-46. 
Anziani OS, Muchiut S. 2014. Resistencia antihelmíntica múltiple (closantel, febendazole, ivermectina y levamisol) en Haemonchus spp. parasitando a ovinos en la provincia de Santa Fe. Ineficacia de una triple combinación de estas drogas para su control. Rev. Med. Vet. (Buenos Aires, Argentina) 95: 22-27.

Besier B. 2007. New anthelmintics for livestock: the time is right. Trends in Parasitol. 23: 21-24.

Cabaret J, Berrag B. 2004. Faecal egg count reduction test for assessing anthelmintic efficacy: average versus individually based estimations. Vet. Parasitol. 121: 105-113.

Cintra MCR, Teixeira VN, Nascimento LV, Sotomaior CS. 2016. Lack of efficacy of monepantel against Trichostrongylus colubriformis in sheep in Brazil. Vet. Parasitol. 216: 4-6.

Coles GC, Bauer C, Borgsteede FH, Geerts S, Klei TR, Taylor MA, Waller PJ. 1992. World Association for the Advance-ment of Veterinary Parasitology (W.A.A.V.P.) methods for the detection of anthelmintic resistance in nema-todes of veterinary importance. Vet. Parasitol. 44: 35-44.

Dash KM, Hall E, Barger IA. 1988. The role of arithmetic and geometric mean worm egg counts in fecal egg count reduction tests and in monitoring strategic drenching programs in sheep. Aust. Vet. J. 65:66-68.

Dobson RJ, Barnes EH, Tyrell KL, Hosking BC, Larsen JWA, Besier RB, Love S, Rolfe PF, Bailey JN. 2011. A multispecies model to assess the impact of refugia on worm control and anthelmintic resistance in sheep grazing systems. Aust. Vet. J. 89: 200-208.

Dobson RJ, Hosking BC, Jacobson CL, Cotter JL, Besier RB, Stein PA, Reid SA. 2012. Preserving new anthelmintics: A simple method for estimating faecal egg count reduc-tion test (FECRT) confidence limits when efficacy and/or nematode aggregation is high. Vet. Parasitol. 186:79-92.

Entrocasso C, Alvarez L, Mananzza J, Lifcschitz A, Borda B, Virkel G, Mottier L, Lanusse C. 2008. Clinical efficacy assessment of the albendazole-ivermectin combination in lambs parasitized with resistant nematodes. Vet. Parasitol. 155: 249-256.

Kaminsky R, Rufener L, Bouvier J, Lizundia R, Schorderet Weber S, Sager H. 2013. Worms-A "license to kill". Vet. Parasitol. 195:286-291.

Kaplan RM. 2004. Drug resistance in nematodes of veterinary importance: a status report. Trends in Parasitol. 20:477-481

Kochapakdee S, Pandey, VS, Pralomkarm W, Choldumrongkul S, Ngampongsai W, Lawpetchara A. 1995. Anthelmintic resistance in goat in southern Thailand. Vet. Rec. 137, 124-125.

Leathwick DM, Waghorn TS, Miller CM, Candy PM, Oliver AMB. 2012. Managing anthelmintic resistance - use of a combination anthelmintic and leaving some lambs untreated to slow the development of resistance to ivermectin. Vet. Parasitol. 187: 285-294.

McKenna PB. 2006. Further comparison of faecal egg count reduction test procedures: Sensitivity and specificity. N. Z. Vet. J. 54:365-366
Mederos AE, Ramos Z, Banchero GE. 2014. First report of monepantel Haemonchus contortus resistance on sheep farms in Uruguay. Parasit. Vectors 7:598.

Presidente PJA. 1985. Methods for detection of resistance to anthelmintics. In: Anderson, N., Waller, P.J. (Eds.), Resistance in Nematodes to Anthelmintic Drugs. CSIRO Division of Animal Health, Glebe, NSW, Australia, 13$28 \mathrm{pp}$.

Roberts F y O'Sullivan P. 1949. Methods for egg counts and larval culture for strongyles infesting gastrointestinal tract of cattle. Aust. J. Agric. Res. 1: 99-102.

Romero JR, Sánchez R, Boero C. 2007. Nematodes. Epidemiología y control. Epidemiología de la gastroenteritis verminosa de los ovinos en la pampa húmeda y la mesopotámica. En: Enfermedades parasitarias de los ovinos y otros rumiantes menores en el cono sur de América. Suárez V, Olaechea $F$, Romero J y Rossanigo C. INTA. Publicación Técnica N. 70, ISSN 0325-2132, 33-42 pp.

Romero JR, Anziani OS, Cetra B, Fiel CA. 2013. Epidemiologia e impacto productivo de nematodes gastrointestinales en la región NEA. En: Enfermedades Parasitarias de Importancia Clínica y Productiva en Rumiantes. Fundamentos epidemiológicos para su diagnóstico y control. Fiel C y Nari A. Ed: Agropecuaria Hemisferio Sur SRL. Uruguay. 89-112 pp.

Sales N \& Love S. 2015. Multi-drug including monepantel (Zolvix ${ }^{\circledR}$ ) resistant Haemonchus confirmed in sheep on a farm in Greater Sydney region. https://wormmailinthecloud.wordpress.com/tag/mon epantel-resistance/in-nsw-australia/ (Verificado:23/02/2016)

Scott I, Pomroy WE, Kenyon PR, Smith G, Adlington B \& Moss A. 2013. Lack of efficacy of monepantel against Telador-sagia circumcincta and Trichostrongylus colubriformis. Vet. Parasitol. 198: 166-171.

Steffan P, Sánchez E, Entrocasso C, Fiel C, Lloberás $M$, Riva E, Guzmán M. 2011. Eficacia de monepantel contra nematodes de ovinos con resistencia antihelmíntica múltiple en la Región Templada de Argentina. Vet. Arg. 273

Stein P, Kaminsky R, Wenger A, Mahoney R. 2011. Persistent activity of monepantel against gastrointestinal nemato-des of sheep. N. Z. J. Zool. 38: 126.

Van der Brom R, Moll L, Kappert C, Vellema P. 2015. Haemonchus contortus resistance to monepantel in sheep. Vet. Parasitol. 209: 278-280.

Van Wyk JA. 2001. Refugia- Overlooked as perhaps the most potent factor concerning the development anthelmintic resistance. Vet. Res. 68:55-67.

Waller P. 2003. Global perspectives on nematode parasite control in ruminant livestock: the need to adopt alternatives to chemotherapy, with emphasis on biological control. Anim. Health. Res. Review. 4: 3543. Burkart RN, Bárbaro N, Sánchez R, Gómez D. 1999. Eco-regiones de la Argentina. APN-PRODIA, 43pp. 